\title{
$\widehat{A}$ Madridge
}

madridge Journal of Women's Health and Emancipation

Interconnecting Scientific World

Review Article

Open Access

\section{Intimate Partner Violence among Women and Trauma-Informed Care: An International Perspective}

\author{
Eula W Pines ${ }^{*}$ \\ Professor at Troy University School of Nursing, Alabama, Phenix City Campus, USA
}

\section{Article Info}

*Corresponding author:
Eula W Pines
Professor
Troy University School of Nursing
Troy, Alabama, Phenix City Campus
USA
E-mail: epines@troy.edu

Received: July 31, 2017

Accepted: August 18, 2017

Published: August 24, 2017

Citation: Pines EW. Intimate Partner Violence among Women and Trauma-Informed Care: An International Perspective. Madridge $J$ Womens Health Emancipation. 2017; 1(1): $11-15$

doi: $10.18689 / \mathrm{mjwh}-1000104$

Copyright: @ 2017 The Author(s). This work is licensed under a Creative Commons Attribution 4.0 International License, which permits unrestricted use, distribution, and reproduction in any medium, provided the original work is properly cited.

Published by Madridge Publishers

\begin{abstract}
Purpose: The purpose of this review was to summarize the prevalence of intimate partner violence and resultant consequences on women's health and to describe an evidence-based trauma-informed care model and a gender-trauma-specific group intervention.
\end{abstract}

Method: An integrative literature review of publications was identified through a comprehensive search of relevant databases (Cochrane Systematic Review, Agency for Healthcare Research and Quality, National Guidelines Clearinghouse, PubMed, CINAHL, EMbase) and other relevant databases. Articles were limited to primary clinical research, systematic reviews, and meta-analyses of clinical studies published in English from 2010 to 2017.

Findings: Worldwide, women are disproportionately burdened by intimate partner violence and significant preventable public health issues. Intimate violence is associated with myriad adverse health outcomes for women, including chronic pain, disability, psychological trauma, neurological injuries, physical and psychological sequelae, and infectious diseases. The World Health Organization estimated that, globally, 35\% of women living in industrialized and developed countries have experienced exposure to physical and/or sexual intimate partner violence. Nurses and other health providers are in key positions to respond to women's trauma associated with intimate partner violence. Evidence suggests that trauma-informed care and trauma-specific interventions such as the trauma recovery empowerment model are effective strategies to strengthen women's healing and recovery.

Conclusion: Intimate partner violence is a worldwide pervasive public health problem. The prevalence rates and myriad adverse consequences on women's health require nurses and other health care providers to employ trauma-informed care and traumaspecific interventions. Additionally, policy making and future research are paramount.

Keywords: Women and trauma; women intimate partner violence and women and coping; intimate partner violence and trauma-informed care and interventions

\section{Introduction}

Worldwide, women are disproportionately burdened by intimate partner violence (IPV) and significant preventable public health problems [1-4]. IPV is associated with myriad adverse health outcomes for women, including chronic pain [5], disability [6], psychological trauma, neurological injuries, physical and psychological sequelae [7-15], and infectious diseases [16]. The World Health Organization (WHO) [2] estimated that, globally, 35\% of women living in industrialized and developed countries have experienced exposure to physical and/or sexual IPV. The percentage of women assaulted 
by intimate partners varies; however, in 48 population-based surveys from around the world, the WHO noted that $10 \%$ to $69 \%$ of women have reported exposure to violent assaults by an intimate partner [3] and 38\% of female homicides were committed by intimate partners [3]. The Centers for Disease Control and Prevention (CDC) [1, para 1], defines intimate partner violence as "individuals' exposure to physical and sexual violence, stalking, and psychological aggression (including coercive acts) by a current or former intimate partner."Intimate partners are individuals with whom a person has a close personal relationship [2]. The CDC elaborated that relationships may be characterized by emotional connectiveness, regular contact, ongoing physical and/or sexual behaviors, identification as a couple, and familiarity with and knowledge of each other's lives [2]. Despite the enormous influence of IPV borne by women, this problem is often unrecognized by health care providers and increases women's risk for HIV infections and myriad chronic physical and mental health sequelae $[16,1-4]$.

Researchers have acknowledged that some women are violent in relationships with men and that violence is also found in same-gender relationships; however, evidence shows that the strongest risk factor for being a victim of IPV is being female [1-5]. Furthermore, many women seek treatment in adulthood for enduring physical and mental health conditions related to childhood traumatic events [6-11]. The CDC [17, para.1] described a traumatic event as "an event or series of events that causes moderate to severe stress reactions." Traumatic events such as IPV have no end point and may represent a single experience or an enduring or repeating event that overwhelms the individual's ability to cope or to integrate the ideas and emotions involved with that experience [17]. These traumatic events often influence women to seek support from health-care providers, but trauma related to IPV is frequently not identified because of barriers such as health-care providers' insensitivity and women's lack of disclosure [18-21].

Because health-care providers are often the first point of contact when women disclose personal exposure to IPV, nurses and other health-care providers play an important role in assessing, treating, and evaluating women's health outcomes related to this traumatic experience [18-21]. If trauma and IPV are not addressed, many women may inadvertently suffer from mental illness and chronic or infectious diseases, including HIV [5]. Thus, the need to address trauma in this vulnerable population is increasingly viewed as an important component of effective behavioral health care [8]. Despite this need, researchers have found that many health-care providers are unprepared to address trauma linked to IPV in women $[4,5,7,8]$. Consequently, if a trauma is unaddressed, traumatic events such as IPV can contribute to life-long adverse health outcomes in women's lives $[2,20]$.

The purpose of this review is to summarize the prevalence of IPV and the effects of IPV on women's health. Additionally, the author will present an evidenced-based trauma-informed care (TIC) model for adaptation in organizational and healthcare practice settings.

\section{Methods}

Findings from relevant peer-reviewed articles from relevant databases such as Cochranane, CINAHL, PubMed, Psycho Review, and other databases were reviewed. Keywords included the following: women and trauma; IPV; IPV and coping; traumatic stress; coping; and trauma-informed care. The term trauma in this article depicts series of events or sets of circumstances that are experienced and perceived by an individual as physically or emotionally harmful or lifethreatening and that have lasting adverse effects on the individual's functioning and mental, physical, social, emotional, or spiritual well-being [22]. Articles are limited to primary clinical research, systematic reviews, and meta-analyses of clinical studies published in English between 2010 and 2017. This author is sensitive to trauma experienced by men's exposure to IPV, but this paper focuses on highlighting the myriad adverse consequences to women who have faced IPV. Articles were excluded if trauma focused primarily on men and children, except when the articles linked adverse childhood experiences (ACEs) to IPV and adult chronic diseases.

\section{Violence against Women and Women's Health}

Violence against women within partner relationships takes many forms: physical, sexual, psychological, and economic. However, IPV is the most common form [3]. These forms of violence are interrelated and affect women from before birth to old age [2-5]. In 2017, the CDC published the National Intimate Partner and Sexual Violence Survey (NISVS): 2010-2012 State Report [23]. Key findings revealed that, in the United States, approximately 1 in 3 women and 1 in 6 men have experienced some form of contact violence during their lifetime. The report underscored the impact of violence on women. A closer look at the report showed that 23 million women compared to 1.7 million men have been victims of completed or attempted rape [22]. Additionally, more than $27 \%$ of women compared with $11 \%$ of men have experienced contact sexual violence, physical violence, and/or stalking by partners and intimate partner violence; and 1 in 6 women and 1 in 19 men experienced stalking during their lifetime, respectively [23]. Negative health responses to IPV include the following: gastrointestinal disorders, substance abuse, sexually transmitted diseases, HIV, and gynecological or pregnancy complications requiring hospitalizations [23]. Survivors also experienced maladaptive psychological responses such as anxiety, depression, substance abuse, suicidal behaviors, and low self-esteem $[5,20]$.

Similarly, in Spain, legal institutions have documented increased IPV recidivism rates against women. Lopez-Ossorio et al., [24] found that in 2015, 129,193 IPV complaints were documented, representing $1.98 \%$ higher IPV reporting rates than in 2014 [6]. Other survey data revealed that $10.3 \%$ of women 16 years or older have suffered physical violence perpetrated by current or former partners, $81.1 \%$ experienced sexual violence, and $25.4 \%$ have endured psychological violence sometimes in their lives [24]. 
Australian researchers Loston et al., [5] conducted a 16year longitudal randomized cohort study to determine the impact of IPV on women's mental and physical health across three generations. The participants comprised 40,395 women representing three cohorts. The findings showed all cohorts living with intimate partners were more likely to report poorer mental health, physical decline in general health and function, and overall higher levels of somatic pain compared to the general population [25].

Machisa et al., [26] surveyed 511 women from Gauteng, South Africa. They found that $50 \%$ experienced IPV in their lifetime and 18\% experienced IPV 12 months before the survey. Overall, $23 \%$ were depressed, $14 \%$ engaged in binge drinking, and $11.6 \%$ experienced post-traumatic stress disorder symptoms. Moreover, $86 \%$ of the women had experienced child abuse. They concluded that mental illness plays a mediating role in the relationship between child abuse and recent IPV. However, IPV negatively affected women's mental health [26]. The researchers asserted that effective integration of mental health services in primary care, detection of IPV symptoms, brief intervention, and broad referral mechanisms for community-based care are necessary to meet the needs of victims of IPV. The researchers elaborated that programs designed to assist abused children need to take similar approaches to reduce the long-term mental effects associated with violence exposure [26].

Traumatic events such as IPV affect a woman's inner and outer self [27]. When trauma affects the inner self, it impacts women's thoughts, feelings, beliefs, and values, resulting in the, and disability in adulthood after controlling for important confounders [30].

Additionally, many researchers have asserted that exposure to trauma predisposes a woman to alcohol and substance abuse [31]. According to Gilbert et al., [32] approximately $80 \%$ of women who seek treatment for substance use disorders have high lifetime prevalence rates of physical or sexual trauma.

Many women may feel that the world is unsafe and that others should not be trusted. Trauma can also affect a woman's outer self, which includes relationships and behaviors. Many women who have experienced trauma struggle with interpersonal relationships with family, friends, and sexual partners. Their parenting skills are challenged, particularly when a woman has experienced childhood trauma [27].

A landmark ACEs [28] study has linked childhood traumatic experiences to domestic violence and IPV, resulting in long-term chronic physical and mental health outcomes in men and women. The findings also indicated that, compared with men, women were more vulnerable to childhood sexual abuse and revictimization. Similarly, Brown et al., [29] collected baseline survey data on health behaviors, health status, and exposure to ACEs, from 17,337 adults aged greater than 18 years and found a positive correlation between ACEs and risk of premature death.

Campbell et al., [30] analyzed 49,526, US adults from 5 states in the 2011 using the Behavioral Risk Factor Surveillance System. Exposures included psychological, physical, and sexual forms of abuse, including substance abuse, violence, mental illness, and incarceration. The researchers concluded that, in addition to having a cumulative effect, individual ACE components had complex relationships with risky behaviors, a period.

Overall, cumulative data underscores the burden of IPV on girls and women. Consequently, these adverse effects of IPV on women's physical and mental health provide compelling evidence for health-care providers to provide TIC [33-36] a period.

\section{Trauma-Informed Care}

The Substance Abuse and Mental Health Services Administration (SAMSHA) [22] establishes a TIC framework as an approach to engaging people with histories of trauma; this framework enables people (health care providers and others) to recognize these symptoms and acknowledges the role that trauma has played in individuals' lives [22].

TIC is an organizational change process that focuses on principles to promote healing and reduce the risk for re-traumatization for vulnerable people [34-37]. SAMSHA approached this framework by integrating trauma research findings, practice-generated knowledge related to trauma interventions, and lessons learned from survivors of trauma, including IPV survivors. Bowen and Muurshid argued that many health and social problems are linked to trauma, suggesting TIC is relevant to a broad range of local, state, federal, and international policies [37].

According to SAMSHA, a TIC program, organization, or system realizes the widespread impact of trauma and understands potential pathways for recovery; recognizes the signs and symptoms of trauma in clients, families, staff, and others involved in the system; responds by fully integrating knowledge about trauma into policies, procedures, and practices; and seeks to actively resist re-traumatization [22].

TIC approaches can be implemented in any type of service organization, but clear distinctions are made between $\mathrm{TIC}$ and trauma-informed interventions or treatment. Overall, the overall aim of TIC is to promote healing by addressing the consequences of trauma, in this case IPV.

SAMSHA's TIC approach is grounded by six key principles [37]:

1. Safety. TIC embraces program efforts to ensure service users' physical and emotional safety, including freedom from harm or danger, and to prevent further trauma from occurring. For example, during assessment, if women feel safe, they are more likely to report violence $[22,37]$.

2. Trustworthiness and transparency include the extent to which an organization maintains transparency in its policies and procedures, with the objective of building trust among stakeholders such as women who experience IPV, staff, and community members [22, 37].

3. Peer support is needed to support female survivors in shared decision-making and in establishing safety and hope, building trust, enhancing collaboration [22, 37].

4. Collaboration and mutuality in TIC means that agency staff view service users as active partners and experts in their lives with peer mentors' interprofessional teams $[22,37]$. 
5. Empowerment, voice, and choice include efforts to share in decision-making at individual and agency levels. Having meaningful choices and options gives female survivors a level of control and is associated with positive health outcomes [22, 37].

6. Cultural, historical, and gender issues or intersectionality references awareness of identity characteristics such as race, gender, sexual orientation, and the privileges or oppression these characteristics can incur $[22,37]$.

\section{Trauma-Specific Interventions}

Trauma-specific interventions are based on the following psychosocial educational empowerment principles [38]:

1. The survivors' need to be respected, informed, connected, and hopeful regarding their recovery.

2. The interrelation between trauma and symptoms of trauma such as substance abuse, eating disorders, depression, and anxiety.

3. The need to work in a collaborative way with survivors, family, friends of survivors, and other human services agencies in a manner that will empower survivors and consumers.

For example, the trauma recovery and empowerment model (TREM) is an evidence-based gender-specific group intervention appropriate for women with exposure to IPV. The model is listed on the CDC's website with the compendium of evidence-based specific trauma interventions.

Fallot et al., [39] designed a quasi-experimental study to test the effectiveness of TREM, a group intervention for female trauma survivors, compared with usual care. The study included 250 women with histories of physical and/or sexual abuse and simultaneously occurring serious mental illnesses and substance use disorders. Comprehensive assessments were completed at 6 and 12 months. The researchers found that the TREM participants showed reductions in alcohol intake, drug abuse severity, anxiety symptoms, and current stressful events. Moreover, the participants demonstrated greater increases in perceived safety [39]. Thus, this trauma-specific group intervention was appropriate for women survivors of IPV.

Similarly, in a recent study, researchers found that TREM group psychotherapy may be beneficial for some participants particularly for female trauma survivors with symptoms of dissociation and self-esteem [40].

\section{Conclusion}

IPV is a worldwide pervasive public health problem. The prevalence rates and myriad adverse consequences for women's health require nurses and other health-care providers to demonstrate sensitivity to the needs of this vulnerable population. These evidence-based approaches enable nurses and other health-care providers to empower female survivors of IPV. Additionally, trauma-specific interventions provide the tools for women's empowerment and recovery, thus enabling women to regain a sense of self and, ultimately, self-empowerment.

\section{Conflict of interest}

The authors confirm that there is no conflict of interest regarding this manuscript.

\section{References}

1. Centers for Disease Prevention and Control. Intimate Partner Violence: Definitions, 2016.

2. World Health Organization. Responding to intimate partner violence, 2013.

3. World Health Organization. Violence by intimate partners.

4. United Nations. Violence against women. United Nations SecretaryGeneral Campaign to End Violence against Women, 2017.

5. Taft C, Schwartz S, Liebschutz JM. Intimate partner aggression in primary care chronic pain patients. Violence. Vict. 2010; 25(5): 649-641.

6. Curry MA, Renker P, Robinson-Whelen S, Hughes RB, Swank P. et al., Facilitators and barriers to disclosing abuse among women with disabilities. Violence. Vict. 2011; 26(4): 430-444. doi: 10.1891/0886-6708.26.4.430

7. Wong JYH, Fong DYT, Lai V, Tiwari A. Bridging intimate partner violence and the human brain: A Literature Review. Trauma. Violence. Abuse. 2014 22-33.

8. Wheeler, K. Neurophysiology of trauma. In K. Wheeler. Psychotherapy for the Advanced Practice Psychiatric Nurse. 2nd ed. St. Louis, MO: Springer Publishing Company; 2014: 51-93.

9. Perese, EF. Stress-related responses: adaptive behaviors, bereavement, and adjustment disorder, In EF Perese. Psychiatric Advanced Practice Nursing: A Biopsychosocial Foundation for Practice. Philadelphia, PA: FA Davis Company; 2012: 245-267.

10. McEwen BS. Protection and damage from acute and chronic stress: allostasis and allostatic overload and relevance to the pathophysiology of psychiatric disorders. Ann. N. Y. Acad. Sci. 2004; 1032: 1-7. doi: 10.1196/ annals. 1314.001

11. McEwen BS. Allostasis and Epigenetics of Brain and Body Health Over the Life Course: The Brain on Stress. JAMA. Psychiatry. 2017; 74(6): 551-552. doi: 10.1001/jamapsychiatry.2017.0270

12. McEwen BS. Protective and damaging effects of stress mediators: central role of the brain. Dialogues. Clin. Neurosci. 2006; 8(4): 367-381.

13. Aupperle RL, Stillman, $A N$, Simmons $A N$, et al. Intimate Partner Violence PTSD And Neural Correlates of Inhibition. J. Trauma. Stress. 2016; 29(1): 33-40. doi: 10.1002/jts22068

14. Aupperle RL, Melrose AJ, Stein MB, Paulus MP. Executive function and PTSD:disengaging from trauma. Neuropharmacology. 2012; 62(2): 686694. doi: 10.1016/j.neuropharm.2011.02.008

15. Griffing $S$, Lewis CS, Chu M, Sage RE, Madry L, Primm BJ. Exposure to interpersonal violence as a predictor of PTSD Symptomatology Domestic Violence Survivors. J. Interpers. Violence. 2006.

16. Hunnicutt $G$, Lindgreen $K$, Murray C, Olsen L. The Intersection of Intimate Partner Violence and Traumatic Brain Injury: A Call for Interdisciplinary Research. J. Fam. Violence. 2017; 32(5): 411-480. doi: 10.1007/s10896-0169854-7

17. Brezing $C$, Ferrara M, Freudenreich O. The syndemic illness of HIV and trauma: implication for a trauma-informed model of care. Psychosomatics. 2015; 56(2): 107-118. doi: 10.1016/j.psym.2014.10.006

18. Centers for Disease Control and Prevention. Coping with atraumatic event. 2017.

19. Hall A, McKenna B, Dearie V, Maquire T, Charleston R, Furness T. Educating emergency department nurses about trauma-informed care for people presenting with mental health crisis: a pilot study. BMC. Nursing. 2016; 15(21): 1-8. doi:10.1186/s12912-016-0141-y

20. Hegarty K, Tarzial L, Taft A. Interventions to support recovery after domestic and sexual violence in primary care. Int. Rev. Psychiatry. 2016; 28(5): 519-532. doi: 10.1080/09540261.2016.1210103 
21. Kalra N, Tarzia L, Taft A. Training healthcare providers to respond to intimate partner violence against women. Cochrane. Library. 2017. doi: 10.1002/14651858.CD012423

22. Substance Abuse and Mental Health Services Administration. SAMHSA's concept of trauma and guidance for a trauma-informed approach. 2017.

23. Smith SG, Chen J, Basile KC, et al. The National Intimate Partner and Sexual Violence Survey (NISVS). 2017; 2010-2012. State Report. 2017 at the Centers for Disease Control and Prevention, National Center for Injury Prevention and Control: 2017.

24. Lopez-Ossorio JJ, Alvarez JLG, Pascual SB, Garcia LF, Buela-Casal G. Risk factors related to intimate partner violence police recidivism in Spain. Int. J. Clinical. Health. Psychol. 2017; 17(2): 107-119. doi: 10.1016/j.jijchp.2016.12.001

25. Loston $D$, Doija $X$, Andersen $A E$, Townsend $N$. Intimate partner violence adversely impacts health over 16 years and across generations: a longitudinal cohort study. PLoS. One. 2017; 12(6): e0178138. doi: 10.1371/ journal.pone.0178138

26. Machisa MT, Christofides N, Jewkes R. Mental ill health in structural pathways to women's experiences of intimate partner violence. PLOS. One. 2017; 12(4): e0175240. doi: 10.1371/journal.pone.0175240

27. Covington, SS. Beyond Trauma: A Healing Journey for Women: Facilitator's Guide. Center City, MN: Hazelton; 2003.

28. Feli VJ, Anda RF, Nordenberg D, et al. Relationship of childhood abuse and household dysfunction to many of the leading causes of death in adults. The adverse childhood experience (ACE study). Am. J. Prev. Med. 1998; 14(4): 245-58.

29. Brown DW, Anda RF, Tiemeier $\mathrm{H}$, et al. Adverse childhood experiences and the risk of premature mortality. Am. J. Prev. Med. 2009; 37(15): 389-396. doi: 10.1016/j.amepre.2009.06.021.

30. Campbell JA, Walker RJ, Egede LE. Associations Between Adverse Childhood Experiences, High Risk Behaviors and Mordidity in Adulthood. Amer. J. Prev. Med. 2016; 50(3): 344-352. doi: 10.1016/j.amepre.2015.07.022
31. Maniglio, R. The impact of child sexual abuse on health: a systematic review of reviews. Clin. Psychol. Rev. 2009; 29(7): 647-657. doi: 10.1016/j. cpr.2009.08.003

32. Gilbert LK, Breiding MJ, Merrik MT et al. Childhood adversity and adult chronic disease: an update from ten states and the District of Columbia, 2010. Am. J. Prev. Med. 2015; 48(3): 345-349. doi: 10.1016/j. amepre.2014.09.00

33. Devries KM, Mak JY, Child JC, et al. Childhood sexual abuse and suicidal behavior: a metal-analysis. Pediatrics. 2014; 133(5): e1331-44. doi: 10.1542/peds.2013-2166

34. Cleary $M$, Hungerford $C$. Trauma-informed care and the Research Literature: How Can the Mental Health Nurse Take the Lead to Support Women Who Have Survived Sexual Assault? Issues. Ment. Health. Nurs. 2015; 36(5): 370-378. doi: 10.3109/01612840.2015.1009661

35. Reeves E. A synthesis of the literature on trauma-informed care. Issues. Mental.Health.Nurs.2015;36(9):698-709.doi:10.3109/01612840.2015.1025319

36. Milles KL. The importance of providing trauma-informed care in alcohol and other drug services. Drug. Alcohol. Rev. 2015; 34(3): 231-233. doi:.10.1111/dar.12273.

37. Bowen EA, Murshid NS. Trauma-Informed Social Policy: A Conceptual Framework for Policy Analysis and Advocacy. AM. J. Public. Health. 2016 106(2): 223-229. doi: 10.2105/AJPH.2015.302970

38. Substance Abuse and Mental Health Services Administration. SAMHSA's trauma specific interventions 2015.

39. Fallot RD, McHugo GJ, Harris M, Xie H. The Trauma Recovery and Empowerment Model: A Quasi- Experimental Effectiveness Study. J. Dual. Diagn. 2011; 7(1-2): 74-89. doi: 10.1080/15504263.2011.566056.

40. Karatzias T, Ferguson S, Gullone A, Cosgrove K. Group psychotherapy for female adult survivors of interpersonal psychological trauma: a preliminary study in Scotland. J. Ment. Health. 2016; 25(6): 512-519. doi: 10.3109/09638237.2016.1139062 